\title{
Semantic Browsing with PowerMagpie
}

\author{
Laurian Gridinoc, Marta Sabou, Mathieu d'Aquin, \\ Martin Dzbor, and Enrico Motta \\ Knowledge Media Institute (KMi), The Open University, Milton Keynes, UK \\ \{1.gridinoc,r.m.sabou, m.daquin,m.dzbor, e.motta\}@open.ac.uk
}

\begin{abstract}
PowerMagpie is a tool that brings semantic interpretation to classical web pages by dynamically-i.e. during browsing - selecting and making use of a wide range of online available ontologies. We introduce the idea of extending browsing through semantic, ontology-based interpretation. Then, we provide a brief description of the architecture. In the end we underline which aspects of the available online semantic data are demonstrated, what the user may learn and which are the future directions.
\end{abstract}

\section{Introduction}

The web supports a large array of interactions and tasks by providing a scalable infrastructure which makes it possible to locate, explore and exchange knowledge. However, not much support is available for the interpretation of web pages [2], beyond what is provided by the author of each particular resource. Generally speaking the web offers two mechanisms to help users make sense of a resource. The first is hypertext linking, that is normally used to indicate additional explicative material, which can help the user in understanding web content. The second is the Related Pages feature provided by several search engines and directories. While these mechanisms can be useful in many cases, they nevertheless suffer from limitations.

Hypertext linking is provided by the author of a web page, who may have a particular audience in mind or may not be aware of other relevant material. This mechanism cannot be very robust with respect to the generation of new information, which may in principle be useful to interpret earlier resources as few resource providers will keep resources up to date. The Related Pages facility improves on the static hypertext linking, as it dynamically identifies interesting relevant pages and therefore supports a user in exploring a domain and even in moving across domains. However, from the point of view of supporting sensemaking, this feature is not very precise and effective, given that the linking is based on lexical similarity and that typically the search space the user needs to explore explodes very quickly, in sharp contrast to the targeted manner in which page designers insert hyperlinks in web resources.

S. Bechhofer et al.(Eds.): ESWC 2008, LNCS 5021, pp. 802 806 2008.

(C) Springer-Verlag Berlin Heidelberg 2008 


\subsection{Motivation}

Semantic layering (the realisation of semantic links, in addition to the existing syntactic ones) is a notion that was introduced in connection with browsing and navigation in early papers about Magpie 3. Magpie finds and highlights the entities from a particular ontology in the current web page. A pre-condition of a successful parsing within the Magpie-enabled browser is the specification of an ontology-derived lexicon which is used to automatically associating a semantic layer to a web resource. This Magpie-mediated association between an ontology and a web resource provides an interpretative viewpoint or context over the resource in question. While Magpie has been successfully used in a number of applications in the domains of Climate Science, Agriculture, and Academic Research, it requires the user to select a priori which ontology has to be used. Hence, it suffers from the brittleness that typically affects knowledge-based systems: while it works well within a well-defined domain, as soon as the browsing session moves away from the original domain, the support declines dramatically.

A number of other approaches can be found in the literature. However, most of them, when not extracting data from the pages and offering it out of context in a different interface (PiggyBank [4]), tend to deal with structured content, e.g. Sifter [5], and pay no attention to the unstructured web documents. Other tools, which bring in the same interface semantic data within the web page, rely mostly on collaborative annotation, like Trailblazer [7. While this approach can work for well defined communities in well defined domains, it is nevertheless brittlethe quality of the interpretation support relies on the availability and quality of annotation. Hence, there is the need for a tool which is able to dynamically bring relevant semantic information into a browsing session, without being limited to one particular ontology. The goal of PowerMagpie is to bring to the user, opportunistically, the appropriate semantic information relevant to his current information needs, in principle from any ontology available on the web.

\section{Architecture}

From a user perspective, PowerMagpie is an extension of a classical web browser, but the main functionalities it provides are actually realized by server-side software that can be seen as a back-end for the whole architecture. In this way, PowerMagpie is particularly easy to install, as it does not require particular software except a JavaScript enabled web browser.

The PowerMagpie server is the central element of the architecture: it is in charge of realising term extraction, ranking and ontology selection and to transmit the results to the browser extension. The term extraction and ranking are done via a hybrid $\mathrm{TF}^{*}$ IDF algorithm [6] which weights each term against its popularity in a search engine (Google or Yahoo). The top ranked terms represents a lexical document signature [8] and those will be used to bootstrap ontology selection. Watson [1] - an ontology search engine-which makes it possible to access large scale semantic content with the degree of efficiency that is required 


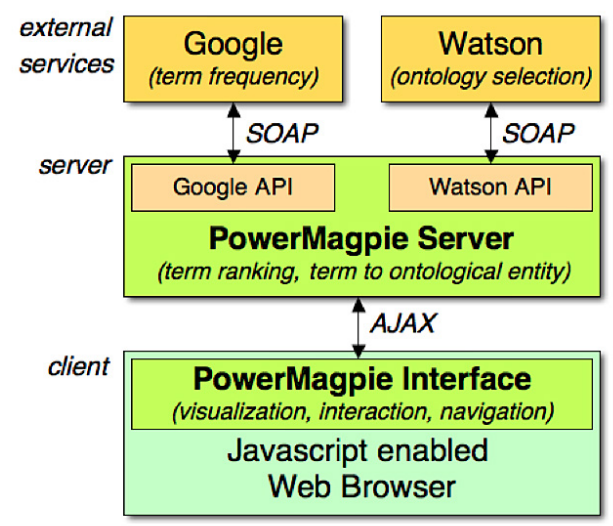

Fig. 1. PowerMagpie architecture

for open semantic browsing. Finally, the interface for the user is in charge of realising the navigation of the semantic and textual information. It is the only part of the architecture that is required to be installed on the client-side, i.e. on the user's computer. The advantage of relying on a common server is that the information computed by the PowerMagpie server can be cached, improving the performance for all the users, based on each individual use of PowerMagpie.

\section{Demonstration}

In our demonstration we will show various functionalities of the tool (i.e., extracting semantic data, navigating the semantic data through the two panels provided - ontology and entity view), applied on web pages of various content while underlying the role of each component within the architecture.

From a user perspective, PowerMagpie is an extension of a classical web browser and takes the form of a narrow, vertical widget displayed on top of the currently browsed web page. This widget provides several functionalities that allow exploring relevant semantic information. A first view, displayed under the Entities panel, lists the key terms from the web page, ordered according to their importance to the document. Each term can be extended to display the semantic information (i.e., ontological entities) it is attached to and allows navigating to the text snippet from where it was extracted. A second, ontology centric view is shown in the Ontologies panel and displays all the ontologies that were identified as relevant for the page. Each ontology can be extended to explore its semantic entities that are relevant for the web page. While the navigation from the ontological entities to the text is a powerful tool for apprehending the document from a semantic perspective, navigating from text to ontologies is important in a situation in which the user needs a semantic definition of a term, either because he is unfamiliar with it or because additional information is needed. This functionality is provided as follows: whenever a word in the text 


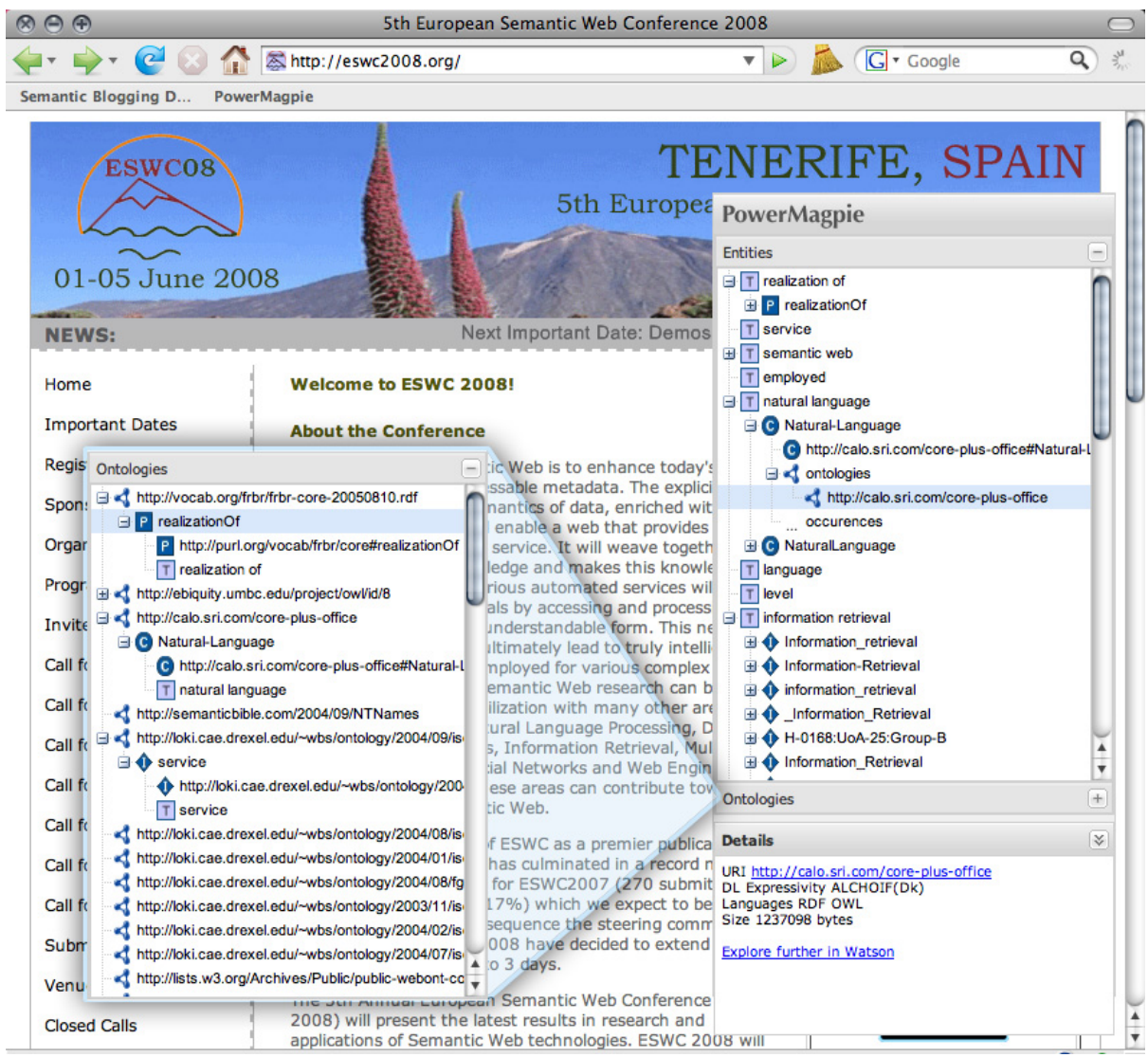

Fig. 2. PowerMagpie screenshot with entity panel in view, ontology panel detailed

of the web page is double-clicked, the PowerMagpie interface moves to the entity panel, to display the semantic description of this term. If the given word has not been already recognised as a key term, then it is automatically added, and the relevant ontologies and ontological entities dynamically selected and explored.

The user will learn - by watching the demo and using the tool himself-how to acquire relevant semantic information and how to make use of it in order to support the sense making process. Because the semantic information is brought in from online available ontologies (via Watson), the user will get a glimpse of the quality of online available data. Further, he will have the chance to use one of the first applications that exploit the semantic web at runtime and experience the added value of such a direct interaction.

A demo of PowerMagpie and a demonstrative video are available at http://powermagpie.open.ac.uk/. 


\section{Future Work}

As the semantic information we can align with the document may be too abstract and of little value to a user which is not a knowledge engineer, we plan to use this information to expand the computed lexical signature of the document to a semantic one - using it as a query expansion approach with a classical lexical search engine (Google or Yahoo) in order to discover semantically related pages. Since query expansion yields high recall and low precision, we will have to filter those results by using the already discovered ontologies in order to validate which of the discovered documents are relevant with the current domain.

We also have to address the presentation of the related semantic information which is cryptic to the regular user by emphasising relations between entities and group them by provenance rather than just displaying URIs as they were intended to be opaque. Regarding the semantically related pages upcoming feature, we intend to address from the previewing of such information to the extent that this information existence may decide which semantic information will be shown to the user, as ontologies that will just define and not 'lead' anywhere would be of little value in semantic browsing.

\section{References}

1. d'Aquin, M., Sabou, M., Dzbor, M., Baldassarre, C., Gridinoc, L., Angeletou, S., Motta, E.: Watson: A Gateway for the Semantic Web. In: Proc. of European Semantic Web Conference, ESWC, Poster Session (2007)

2. Domingue, J., Dzbor, M.: Magpie: supporting browsing and navigation on the semantic web. In: Proceedings of the 9th international conference on Intelligent user interface, pp. 191-197 (2004)

3. Domingue, J., Dzbor, M., Motta, E.: Semantic Layering with Magpie. In: Staab, S., Studer, R. (eds.) Handbook on Ontologies in Information Systems, Springer, Heidelberg (2003)

4. Huynh, D., Mazzocchi, S., Karger, D.: Piggy bank: Experience the semantic web inside your web browser (2005)

5. Huynh, D.F., Miller, R.C., Karger, D.R.: Enabling web browsers to augment web sites' filtering and sorting functionalities. In: Proceedings of the 19th annual ACM symposium on User interface software and technology, pp. 125-134 (2006)

6. Manning, C.D., Raghavan, P., Schütze, H.: Introduction to Information Retrieval. Cambridge University Press, Cambridge (to appear, 2008)

7. Johnston, A., Croke, P., Tighe, K.: Using named entities as a basis to share associative trails between semantic desktops. In: Semantic Desktop Workshop (2005)

8. Phelps, T.A., Wilensky, R.: Robust Hyperlinks Cost Just Five Words Each. University of California, Berkeley, Computer Science Division (2000) 